\title{
ARTí́CULO
}

\section{Origen y evolución del giro ciclónico de la Bahía de Campeche, Golfo de México}

Origin and evolution of cyclonic eddy of the Bay of Campeche, Gulf of Mexico

\author{
Miguel Ángel Díaz-Flores ${ }^{1 *}$, David Alberto Salas-de-León ${ }^{2 * *}$ \\ y María Adela Monreal-Gómez ${ }^{2 * * *}$
}

\begin{abstract}
${ }^{1}$ Posgrado en Ciencias del Mar y Limnología, Universidad Nacional Autónoma de México, Av. Universidad 3000, Col. Copilco, Del. Coyoacán, Cd. de México, 04510, México.*diazmig@comunidad.unam.mx

${ }^{2}$ Instituto de Ciencias del Mar y Limnología, Universidad Nacional Autónoma de México, Av. Universidad 3000, Col. Copilco, Del. Coyoacán,Cd. de México, 04510, México.**dsalas@unam.mx, ***monreal@cmarl.unam.mx

Abstract.- The ocean is characterized by a circulation dominated by vortex of different sizes; the vortex play a very important role in the oceanic climate as they transport energy, mass and organisms. Their study is therefore of first importance to understand the different processes that occurring in the seas at different time-space scales. The Bay of Campeche, in the southern Gulf of Mexico, is characterized by a cyclonic circulation. With the aim of identifying the origin and the formation of the cyclonic vortex, data of currents registered in an oceanographic survey and data of sea altimetry obtained with remote sensors were analyzed. From the results, the formation of the cyclonic vortex of the Bay of Campeche depends mainly on the conservation of the potential vorticity.
\end{abstract}

Key words: Potential vorticity, cyclonic eddies, circulation in bays, altimetry

Resumen.- El océano se caracteriza por una circulación dominada por giros de diferentes tamaños; los giros juegan un papel muy importante en el clima oceánico ya que transportan energía, masa y diversos organismos. Su estudio es por tanto de primera importancia para entender los diferentes procesos que ocurren en los mares a diferentes escalas espacio -temporales. La Bahía de Campeche, en el sur del Golfo de México se caracteriza por tener una circulación de tipo ciclónica. Con la finalidad de identificar el origen y la formación del giro ciclónico se analizaron datos de corrientes registradas en una campaña oceanográfica y datos de altimetría del mar obtenidos con sensores remotos. De los resultados obtenidos se deduce que la formación de los giros ciclónicos de la Bahía de Campeche depende principalmente de la conservación de la vorticidad potencial.

Palabras clave: Vorticidad potencial, giros ciclónicos, circulación en bahías, altimetría

\section{INTRODUCCIÓN}

La circulación de gran escala en el Golfo de México está dominada por dos características semi-permanentes, la intensa Corriente de Lazo y un gran giro anticiclónico, los cuales se sitúan en la parte oriental y occidental del golfo, respectivamente (Behringer et al. 1977). El principal mecanismo de forzamiento de la circulación en el golfo es el viento y el transporte asociado al flujo que entra a través del Canal de Yucatán y que sale por el Estrecho de Florida (Vukovich et al. 1979, Hurlburt \& Thompson 1980, Sturges \& Evans 1983, Sturges \& Bozec 2013). En primavera y verano la Corriente de Lazo penetra más hacia el norte, mientras que en otoñoe invierno se repliega hacia Cuba dando origen al gran giro anticiclónico, siendo éste de aproximadamente $300 \mathrm{~km}$ de diámetro, que posterior a su formación se desplaza hacia el oeste (Leipper 1970, Behringer et al. 1977, Maul 1977). En las zonas de las plataformas costeras de Texas-Luisiana, Florida y la Bahía de Campeche se observan giros ciclónicos, estos giros ciclónicos son más pequeños que el gran giro anticiclónico pues alcanzan diámetros máximos de aproximadamente 150 km (Monreal-Gómez \& Salas-de-León 1990). Se ha postulado que la presencia de los giros ciclónicos en la periferia de la Corriente de Lazo está asociada al desprendimiento del gran giro anticiclónico (Vukovich \& Maul 1985). Sin embargo, se ha observado la formación de los giros ciclónicos sin que se presente el desprendimiento del giro anticiclónico; también, se ha propuesto que los giros ciclónicos pueden ser producidos por el choque de los anticiclónicos con la costa oeste del golfo (Hurlburt \& Thompson 1980, Smith 1986, Vidal et al. 1994), debido a la conservación de la vorticidad potencial (Hurlburt \& Thompson 1982, Hamilton et al. 1999, Ohlmann et al. 2001, Villanueva et al. 2002). 
Uno de los giros ciclónicos, el de mayor tamaño en promedio ( $100 \mathrm{~km})$, es el de la Bahía de Campeche, este giro ha sido estudiado por diferentes autores (Cochrane 1963, Nowlin 1972, Molinari et al. 1978, Hurlburt \& Thompson 1980, Monreal-Gómez 1986, Monreal-Gómez \& Salas-deLeón 1990, Velasco-Mendoza 1994, Díaz-Flores 2004, Pérez-Brunius et al. 2013). La Bahía de Campeche, situada en la región sur del Golfo de México (Fig. 1), presenta una circulación ciclónica durante el otoño (Cochrane 1963, Nowlin 1972, Molinari et al. 1978, Monreal-Gómez 1986, MonrealGómez \& Salas-de-León 1990, Velasco-Mendoza 1994, Díaz-Flores 2004). Los datos sinópticos y los mapas de topografía de altura dinámica, muestran el carácter ciclónico de la circulación en esta bahía durante agosto (Nowlin 1972). Diversos mecanismos han sido propuestos para explicar la formación de este giro. Algunos autores lo atribuyen a una consecuencia de la formación del gran giro anticiclónico (Hurlburt \& Thompson 1980). Cochran (1963) considera que la interacción del agua de la surgencia con el Banco de Campeche es lo que forma el giro ya que esta corriente debe conservar su momento angular. Monreal-Gómez (1986) encuentra la formación del giro mediante un modelo numérico de gravedad reducida forzado solo por variaciones en la corriente de Yucatán, lo cual indica que la surgencia de Yucatán no es el único mecanismo de formación. Otros autores consideran que el giro ciclónico es el resultado de la formación del gran giro anticiclónico y de la geometría de la costa (Velasco-Mendoza 1994, Monreal-Gómez \& Salas-de-León 1997).

Parte del agua que entra al Golfo de México por el Canal de Yucatán, se desvía hacia el oeste, sobre la plataforma de Yucatán y el Banco de Campeche, la profundidad en dicha plataforma no rebasa los $200 \mathrm{~m}$, al llegar a la Bahía de Campeche la profundidad cambia hasta más de $3000 \mathrm{~m}$ (Monreal-Gómez \& Salas-de-León 1990). En la presente investigación se postula la hipótesis de que esta diferencia en profundidad es el mecanismo que genera los giros ciclónicos que se observan en la bahía; ya que, se ha observado que cuando una corriente intensa pasa de una zona de mayor profundidad a una de menor profundidad induce la formación de giros anticiclónicos y de forma contraria cuando la corriente va de una de menor profundidad a una de mayor se forman giros ciclónicos, esto por conservación de la vorticidad potencial (McWilliams 1985, Bracco \& Pedlosky 2003, Stewart 2008), es decir la corriente pasa por una zona de menor a mayor profundidad con diferencias del orden de $2800 \mathrm{~m}$ y como consecuencia de la conservación de la vorticidad potencial dicha característica batimétrica induce a la formación de giros ciclónicos. Este proceso es detalladamente explicado por Stewart (2008) tomando como ejemplo una columna de Taylor.

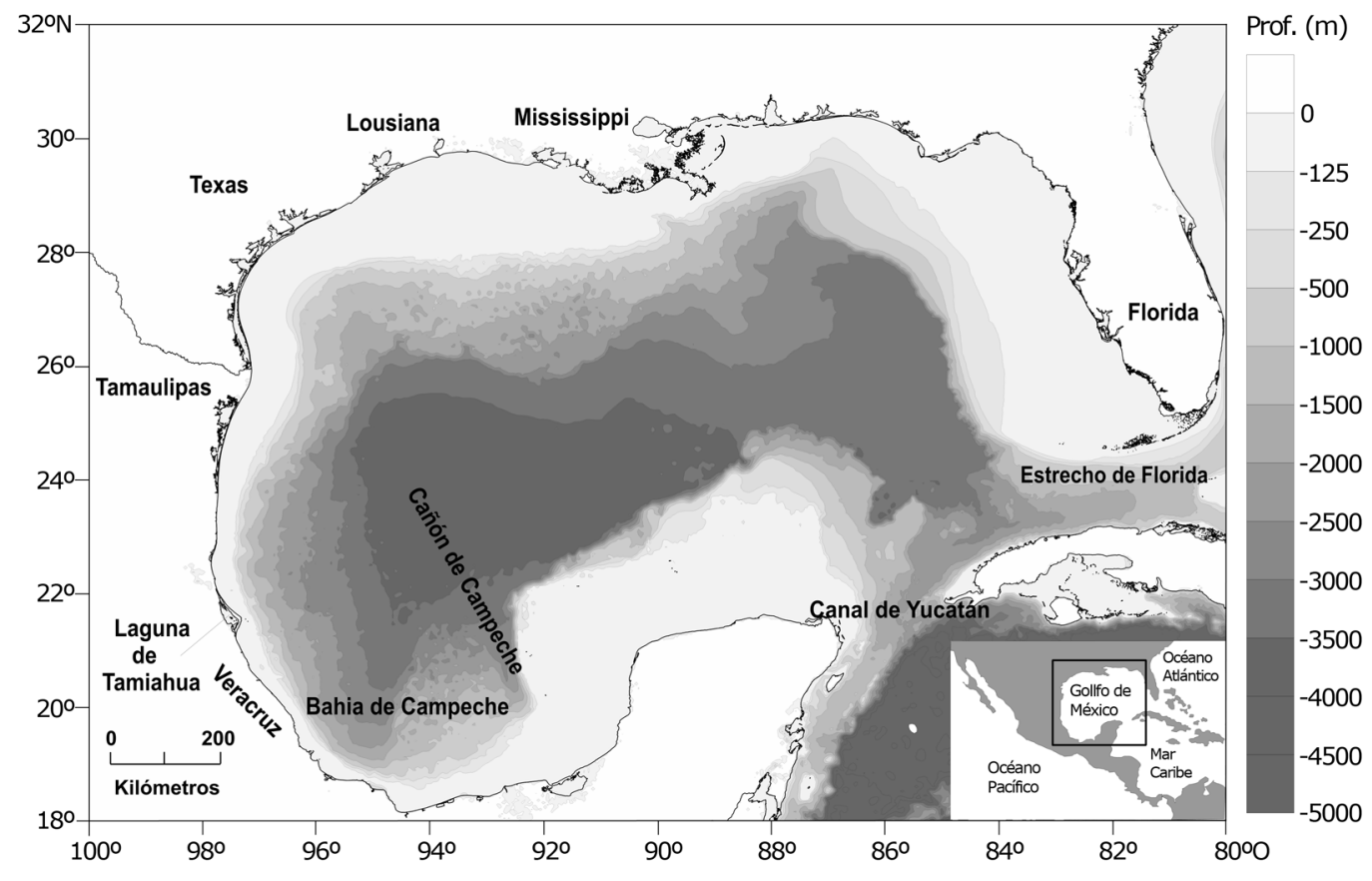

Figura 1. Ubicación del Golfo de México y de la Bahía de Campeche / Location of the Gulf of Mexico and the Bay of Campeche 
Por tanto, el objetivo de este trabajo fue identificar el origen y la formación del giro ciclónico de la Bahía de Campeche, a través de datos de corrientes registrados en una campaña oceanográfica e información de altimetría obtenidos con sensores remotos en satélites en la Bahía de Campeche, sur del Golfo de México.

\section{MATERIALES Y MÉTODOS}

\section{Datos de Campañas oceanográficas}

Se realizó una campaña oceanográfica para la obtención de datos; durante la campaña denominada FIBAC I (Física de la Bahía de Campeche I) llevada a cabo entre el 14 de agosto y 1 de septiembre de 1996 en el buque oceanográfico 'Justo Sierra' de la Universidad Nacional Autónoma de México (UNAM), se realizaron 93 estaciones hidrográficas con un CTD MARK IV. A lo largo de la trayectoria realizada por el buque (Fig. 2), se midieron las corrientes a diferentes profundidades en 128 celdas de $16 \mathrm{~m}$ de espesor cada 0,75 de segundo, con un Perfilador Acústico de Corrientes Doppler (ADCP) de $75 \mathrm{kHz}$, de la compañía RDI. Los datos de las componentes horizontales de la velocidad del agua o corriente $(u, v)$ obtenidos con el ADCP, se registraron a partir de los 4 primeros metros desde la profundidad del transductor del ADCP, esto para quitar el ruido que induce el casco del buque durante la navegación. En promedio, la máxima profundidad de registro confiable fue de $500 \mathrm{~m}$. Los datos se filtraron hasta obtenerlos cada $5 \mathrm{~min}$ equivalente a tener datos de velocidad horizontal en 31 capas de profundidad cada $1800 \mathrm{~m}$ en la horizontal.

\section{INFORMACIÓN DE IMÁGENES DE SATÉLITE}

A partir de imágenes de altimetría de satélite (AVISO, Archiving Validation and Interpretation of Satelite Oceanografic Data), de agosto de 2001 a diciembre de 2007, se realizaron promedios mensuales de altimetría en el Golfo de México con la finalidad de identificar los núcleos de altura negativa, los cuales se asocian a giros fríos o ciclónicos. Con los resultados obtenidos se estimó el tamaño de los diámetros, excentricidad y dirección y rapidez de traslación de los giros. Este análisis se realizó sólo a los giros que permanecieron por más de 2 meses.

\section{VORTICIDAD POTENCIAL}

Con las componentes de la velocidad absoluta horizontal obtenidas con el ADCP, se calculó la componente vertical de la vorticidad relativa $\left(\zeta_{z}\right)$. La vorticidad potencial $(V P)$ está compuesta por la suma de la componente vertical de la vorticidad relativa $\zeta_{z}\left(=\frac{\partial v}{\partial x}-\frac{\partial u}{\partial y}\right)$ más la vorticidad planetaria $f(=2 \Omega \operatorname{sen} \phi)$ dividida por la profundidad (Pond \& Pickard 1978, Knauss 1996, Stewart 2008):

$$
V P=\frac{f+\zeta_{z}}{H}
$$

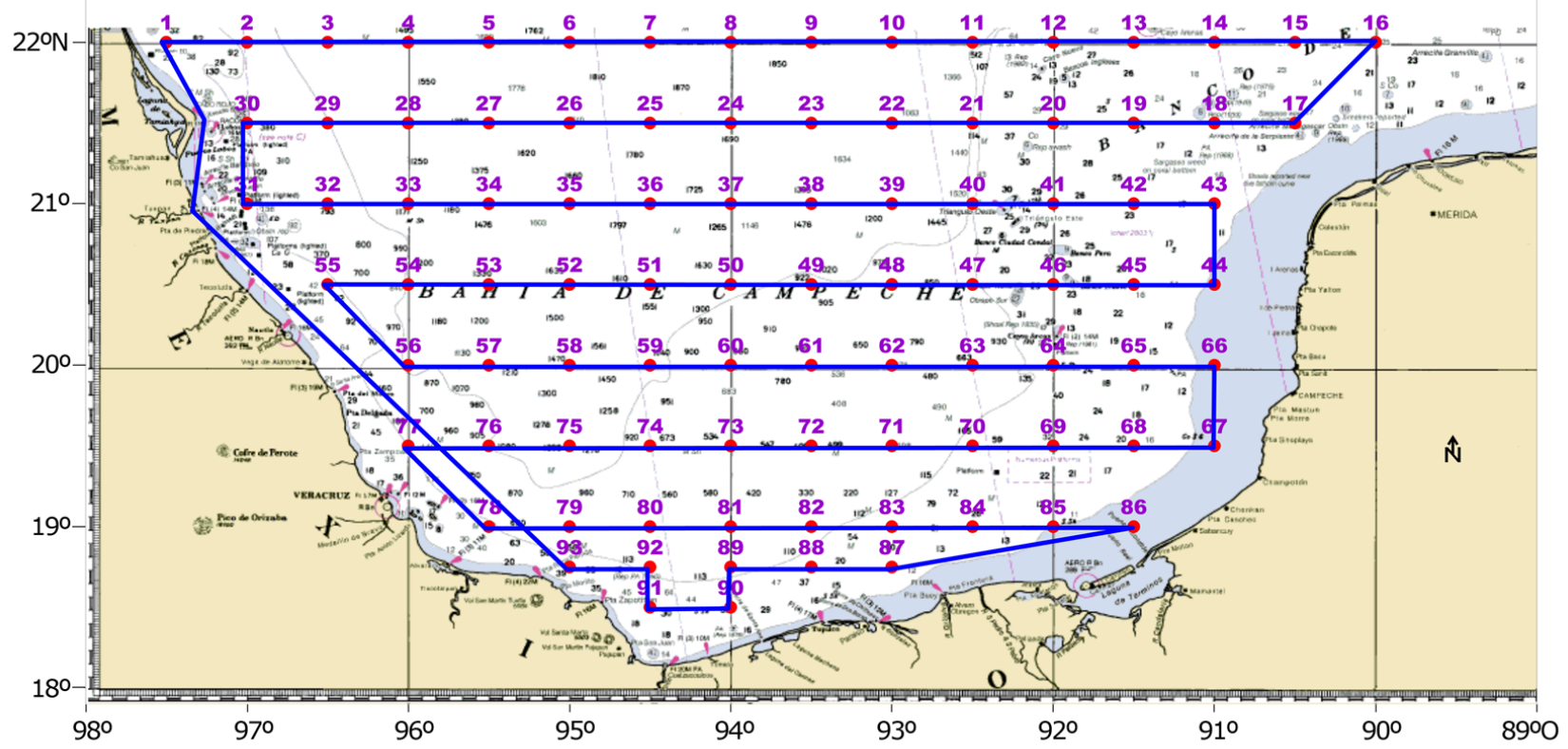

Figura 2. Estaciones hidrográficas obtenidas con CTD (puntos rojos) y trayectoria de registros de corrientes mediante ADCP durante la campaña FIBAC1 (línea azul) / CTD hydrographic stations (red dots) and ADCP current path records during the FIBAC1 cruise (blue line) 
Con los resultados se elaboraron isolíneas de vorticidad relativa correspondientes a las capas de integración de las corrientes de estudio. En el caso del giro de la Bahía de Campeche éste se desplaza en línea casi recta de este a oeste tardándose en promedio 6 meses para ir de la costa este a la oeste de la bahía (el giro inicia su formación en julio y llega a la costa oeste en diciembre (Monreal-Gómez \& Salas-de-León $1990)$, en este caso la vorticidad planetaria $\left(f \sim \mathrm{O}\left(10^{-7}\right)\right)$ es aproximadamente constante y mucho menor que la vorticidad relativa $\left(\zeta_{Z} \sim \mathrm{O}\left(10^{-5}\right)\right)$, por lo que solo se consideró la vorticidad relativa.

\section{Resultados}

Solo se presentan los resultados de las mediciones de velocidades y cálculo de vorticidad en 2 profundidades, capa de los 4 a los 20 m y capa de 84 a $100 \mathrm{~m}$ ya que son los que presentaron mayores diferencias. El patrón de la circulación en la capa de $\operatorname{los} 4$ a los $20 \mathrm{~m}$ de profundidad (Fig. 3), muestra 2 giros ciclónicos con un diámetro aproximado de $140 \mathrm{~km}$ y velocidades promedio de $0,27 \mathrm{~m} \mathrm{~s}^{-1}$. El de mayor diámetro $\left(\mathrm{L}_{1}\right)$ ubicado en $20,20^{\circ} \mathrm{N}$ y $94,23^{\circ} \mathrm{O}$, mientras que el menor $\left(\mathrm{L}_{2}\right)$ en $19,69^{\circ} \mathrm{N}$ y $95,53^{\circ} \mathrm{O}$. Por otro lado, se observa un giro anticiclónico $(\mathrm{H})$ ubicado en $21,00^{\circ} \mathrm{N}-93,23^{\circ} \mathrm{O}$, con velocidades máximas de $0,19 \mathrm{~m} \mathrm{~s}^{-1}$. Se puede observar también, una corriente intensa en la costa oeste de la bahía, hacia el norte con velocidades máximas de $0,43 \mathrm{~m} \mathrm{~s}^{-1}$. En general, el patrón de corrientes en esta capa muestra una circulación ciclónica en la región suroeste de la bahía; así como, la porción sur del gran giro anticiclónico.

Los resultados del cálculo de la componente vertical de la vorticidad relativa para esta capa, muestran valores positivos de hasta $140 \times 10^{-7} \mathrm{~s}^{-1}$ en la región de circulación ciclónica y valores negativos de hasta $-100 \times 10^{-7} \mathrm{~s}^{-1}$ en donde se observa la circulación anticiclónica (Fig. 4). En la región noroeste de la bahía los valores negativos están asociados a la porción sur del giro anticiclónico; así como, en la parte central al norte de la bahía donde se encontró una característica anticiclónica de forma elíptica.

En la capa de los 84 a los 100 m de profundidad se observa una corriente que va hacia el sureste desde $\operatorname{los} 20,00^{\circ} \mathrm{N}$ y $95,70^{\circ} \mathrm{O}$ que sigue la batimetría de la bahía. Hacia el norte de $\operatorname{los} 20,00^{\circ} \mathrm{N}$ en la región próxima a la costa, la corriente se dirige hacia el norte (Fig. 5). Es interesante observar una zona de divergencia en las corrientes, ubicada en $20,00^{\circ} \mathrm{N}$ y $95,70^{\circ} \mathrm{O}$, en este caso indicaría en principio, la región desde donde inicia el ascenso de agua inducido por el giro ciclónico $\mathrm{L}_{2}$.

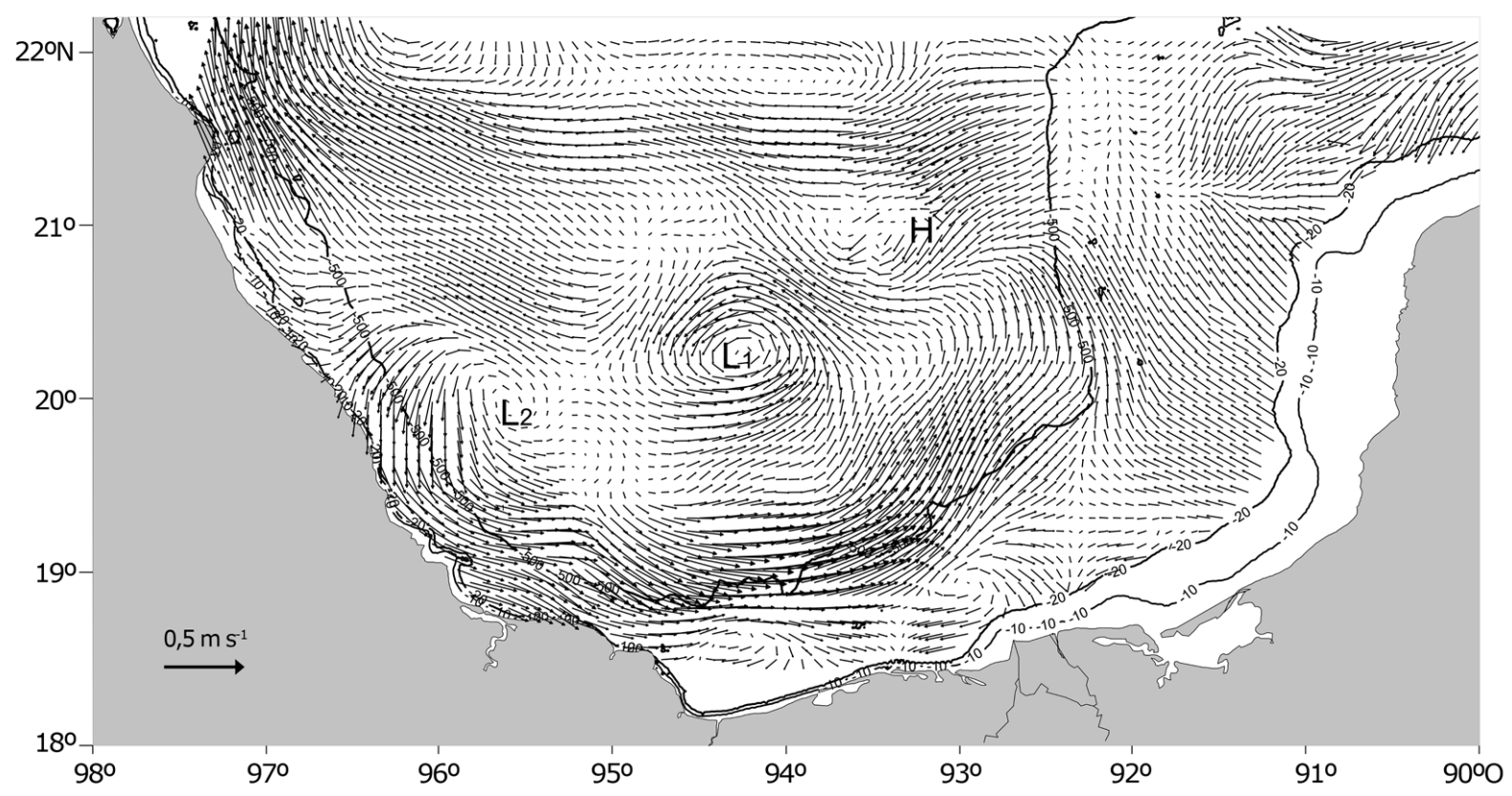

Figura 3. Patrón de circulación ( $\mathrm{m} \mathrm{s}^{-1}$ ) en la capa de 4 a $20 \mathrm{~m}$ de profundidad. $L_{1}$, $L_{2}$ : giros ciclónicos, $\mathrm{H:} \mathrm{giro} \mathrm{anticiclónico} \mathrm{/} \mathrm{Circulation} \mathrm{pattern} \mathrm{(m} \mathrm{s}{ }^{-1}$ ) in the layer 4 to $20 \mathrm{~m}$ depth. $L_{1}, L_{2}$ : cyclonic eddies, $\mathrm{H}$ : anticyclonic eddy 


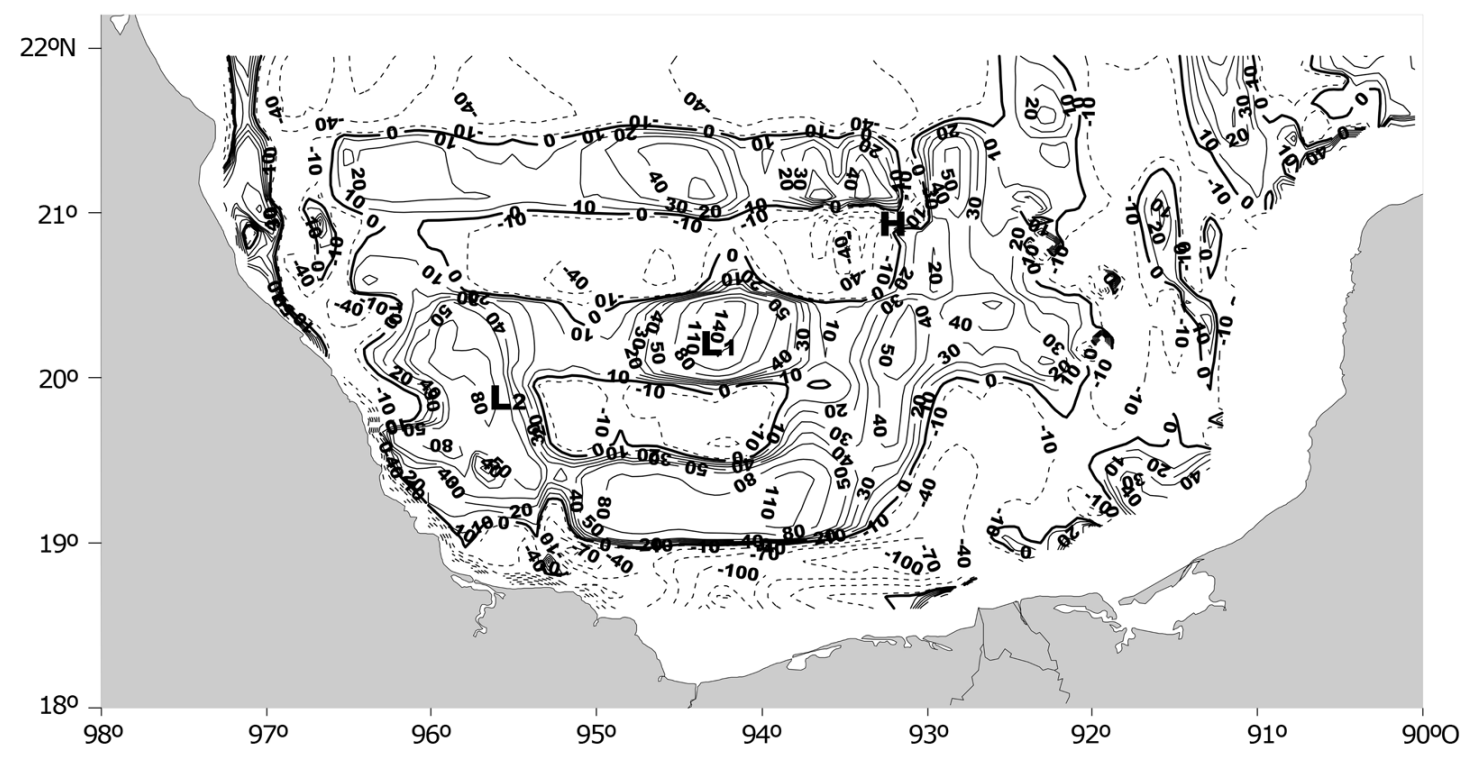

Figura 4. Componente vertical de la vorticidad relativa $\left(\times 10^{-7} \mathrm{~s}^{-1}\right)$ en la capa de 4 a $20 \mathrm{~m}$ de profundidad / Vertical component of the relative vorticity $\left(\times 10^{-7} \mathrm{~s}^{-1}\right)$ at the layer between 4 to $20 \mathrm{~m}$ depth

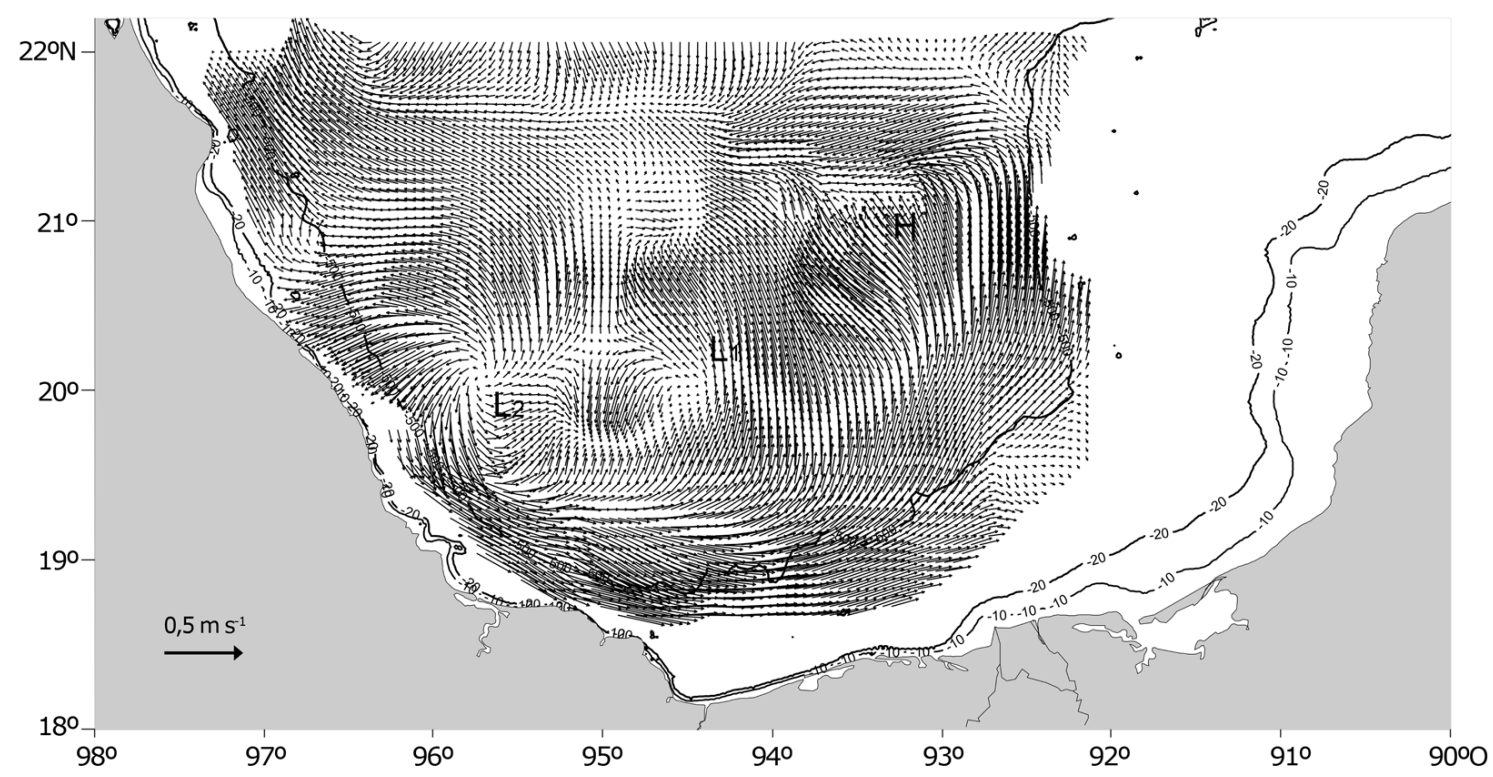

Figura 5. Patrón de circulación ( $\mathrm{m} \mathrm{s}^{-1}$ ) en la capa de 84 a $100 \mathrm{~m}$ de profundidad. $\mathrm{L}_{1}$, $\mathrm{L}_{2}:$ giros ciclónicos, $\mathrm{H:} \mathrm{giro} \mathrm{anticiclónico} \mathrm{/} \mathrm{Circulation} \mathrm{pattern}$ $\left(\mathrm{m} \mathrm{s}^{-1}\right)$ in the layer from 84 to $100 \mathrm{~m}$ depth. $\mathrm{L}_{1}, \mathrm{~L}_{2}$ : cyclonic eddies, $\mathrm{H}$ : anticyclonic eddy 


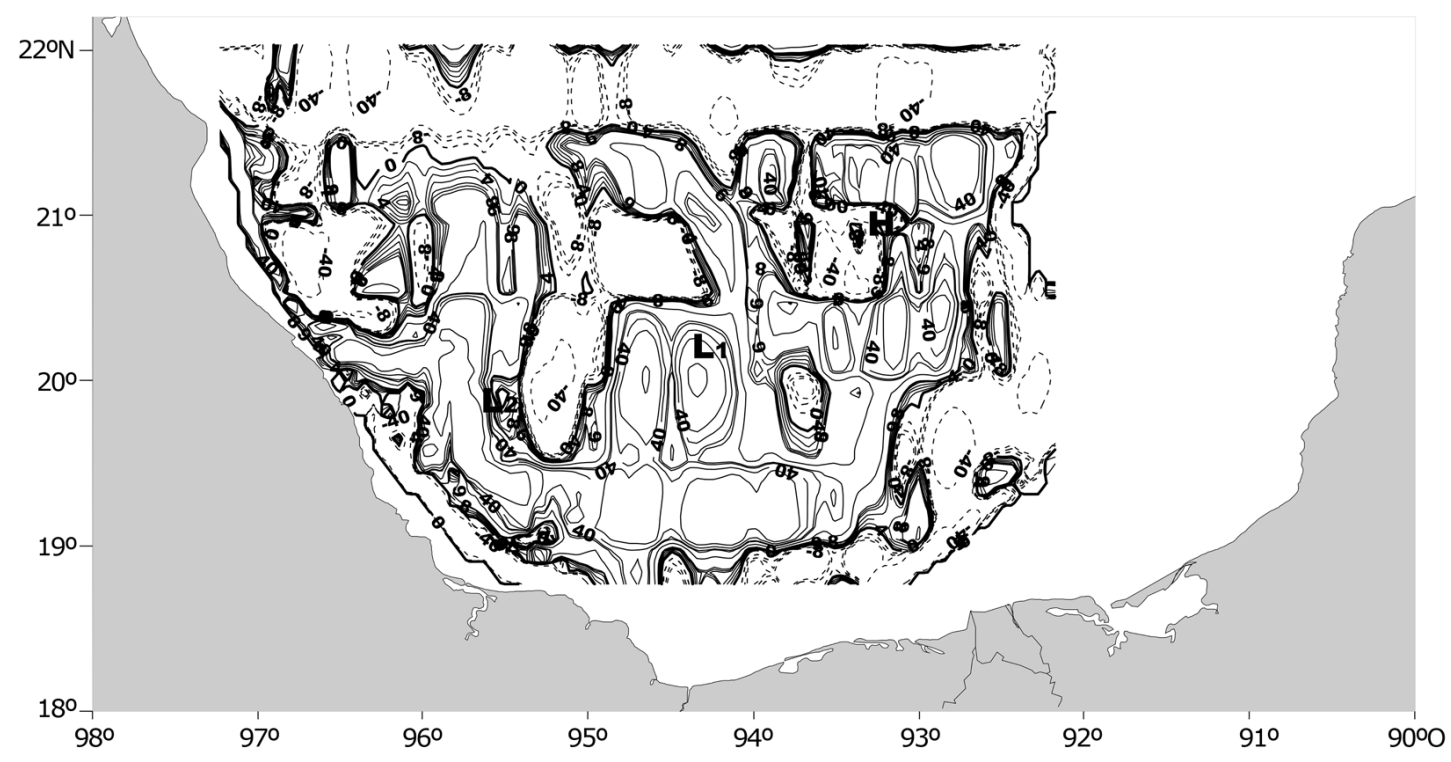

Figura 6. Componente vertical de la vorticidad relativa $\left(10^{-7} \mathrm{~s}^{-1}\right)$ representativo de la capa de 84 a $100 \mathrm{~m}$ de profundidad / Vertical component of the relative vorticity $\left(10^{-7} \mathrm{~s}^{-1}\right)$ in the layer from 84 to $100 \mathrm{~m}$ depth

La corriente en la frontera oeste de la porción noroeste de la bahía, presentó velocidades máximas de $0,29 \mathrm{~m} \mathrm{~s}^{-1}$. La corriente con dirección hacia el oeste (entre $95,00^{\circ}-96,50^{\circ} \mathrm{O}$ y $21,00^{\circ}-22,00^{\circ} \mathrm{N}$ ) es notablemente de menor intensidad que en las capas superiores; además, presenta un patrón de corrientes más uniforme. Esto coincide consecuentemente con una menor vorticidad (Fig. 6). La vorticidad positiva es dominante en la parte central de la bahía y al oeste, mientras que la vorticidad negativa se presenta en forma de elipses alargadas en la costa sureste y en el noroeste indicando una alternancia de circulación ciclónica-anticiclónica.

A partir de imágenes de altimetría se estimó la velocidad promedio de traslación del giro ciclónico ubicado en $94,23^{\circ} \mathrm{O}$ y $20,20^{\circ} \mathrm{N}$, la cual es aproximadamente de $6,1 \mathrm{~km} \mathrm{día}^{-1}$. La trayectoria del centro del giro fue variable, en un principio la dirección de su trayectoria fue hacia el noreste a un rumbo de $85^{\circ}$, después de 4 días fue hacia $35^{\circ}$ y a los 4 días siguientes hacia $74^{\circ}$.

La superficie del mar se deforma por efecto del gradiente de presión generado por la formación de los giros ciclónicos y anticiclónicos, un giro ciclónico como tienen corrientes divergentes mostrará un hundimiento de la superficie del mar, mientras que un giro anticiclónico por ser corrientes convergentes tendrá un ascenso de la superficie del mar. Las imágenes de altimetría y de corrientes medidas con ADCP muestran alta coherencia con los giros ciclónicos y anticiclónicos (Fig. 7)
Tabla 1. Descripción de los giros de mayor diámetro observados en la capa superficial / Description of the larger diameter eddies of observed in the surface layer

\begin{tabular}{|c|c|c|c|c|c|}
\hline \multirow{2}{*}{ Giro } & \multicolumn{2}{|c|}{ Ubicación } & \multirow{2}{*}{$\begin{array}{c}\text { Radio } \\
(\mathrm{km})\end{array}$} & \multirow{2}{*}{$\begin{array}{c}\text { Velocidad } \\
\text { Tangencial } \\
\text { Máxima } \\
\left(\mathrm{m} \mathrm{s}^{-1}\right)\end{array}$} & \multirow{2}{*}{$\begin{array}{l}\text { Velocidad } \\
\text { Máxima } \\
\left(\mathrm{m} \mathrm{s}^{-1}\right)\end{array}$} \\
\hline & Lat. (N) & Long. (O) & & & \\
\hline Ciclónico & 20,20 & 94,23 & 140 & 0,25 & 0,29 \\
\hline Anticiclónico & 21,00 & 94,23 & 80 & 0,19 & 0,19 \\
\hline
\end{tabular}

La Tabla 1 muestra algunas de las principales características de los grandes giros ciclónico y anticiclónico de la Bahía de Campeche. Podemos observar que, en promedio, en la bahía los giros ciclónicos tienen radios y velocidades tangenciales máximas de 1,75 y 1,31 veces mayores respectivamente, que los anticiclónicos.

Por otro lado, el período de rotación del giro ciclónico está dado por $T=\frac{2 \pi r}{u}$, donde $r(140000 \mathrm{~m}$, ver tabla 1$)$ es el radio del giro ciclónico y $u\left(0,25 \mathrm{~m} \mathrm{~s}^{-1}\right)$ la velocidad de la corriente del giro, por lo que $T=40,72$ días que corresponde a un valor típico de vorticidad relativa en el océano ( un ciclo por mes; Stewart 2008). El período inercial está dado por $T_{i}=\frac{2 \pi}{f}$, si el valor del parámetro de Coriolis $f(=2 \Omega \operatorname{sen} \phi)$ para esa latitud es $5.038 \times 10^{-5} \mathrm{~s}^{-1}$, entonces se obtiene un período inercial $\left(T_{i}\right) \mathrm{de}$ 


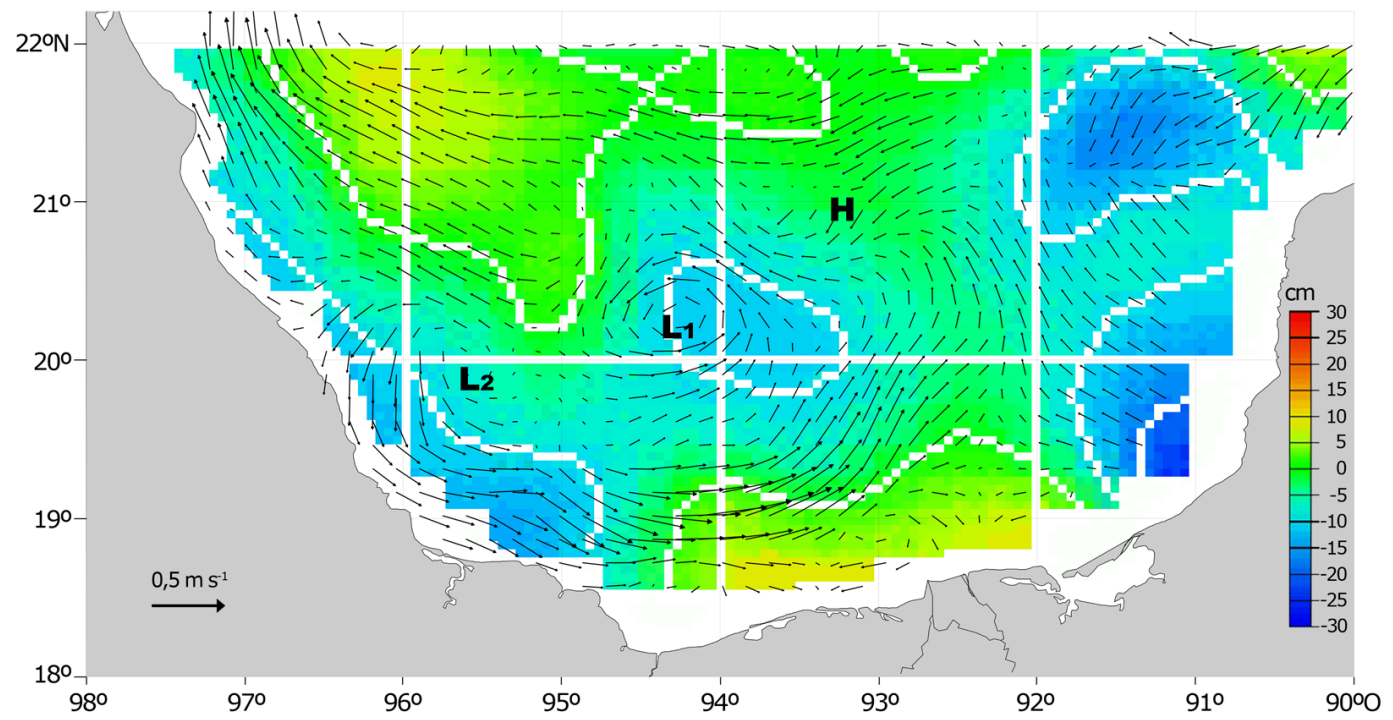

Figura 7. Corrientes obtenidas con el ADCP en la capa de los 4-20 $\mathrm{m}$ y anomalía de altura de la superficie del mar ( $\mathrm{cm}$ ) en la Bahía de Campeche. 1 de septiembre de 1996 বhttp:// www-ccar.colorado.edu/research/alt/html/alt_nrt.html >. L, L: giros ciclónicos, H: giro anticiclónico / ADCP currents in the layer of 4-20 m and sea surface height anomaly $(\mathrm{cm})$ in the Bay of Campeche. September 1, $1996<\mathrm{http}: / /$ www-ccar.colorado.edu/research/ alt/html/alt_nrt.html $>$. $L_{1}, L_{2}$ : cyclonic eddies, $H$ : anticyclonic eddy

1.44 días. Por otro lado, el número de Rossby es definido como un cociente entre dos términos, un término advectivo y el término de la fuerza de Coriolis:

$$
R_{o}=\frac{(d u / d t)}{(u f)} \approx \frac{\left(u^{2} / r\right)}{(u f)}=\frac{(u / r)}{f}=\frac{u}{f r} ;
$$

quedando expresado finalmente como $R_{o}=\frac{u}{f r}$ (Pedlosky 1979) donde $u$ y $r$ serán la velocidad y la longitud característica del giro respectivamente, esto nos da para el giro ciclónico 0,041 . Este número compara los efectos inerciales (cambios locales y efectos advectivos) frente al efecto de Coriolis. Para que los efectos de la rotación terrestre no sean considerados como importantes el número de Rossby debe ser mucho mayor que 1. Por lo tanto, el valor del número de Rossby indica que la aproximación geostrófica es válida y que la aceleración de Coriolis $u$ es mayor que la aceleración horizontal del fluido $\frac{d u}{d t}$; es decir $\frac{d u}{d t}\langle\langle u f$. Este resultado muestra que el giro ciclónico de la Bahía de Campeche, es un proceso de gran escala y no obstante que el período inercial es menor que el rotacional, tienen importancia para los efectos rotacionales de la Tierra. Por otro lado, Las partículas transportadas por las corrientes inerciales describen círculos cuyos diámetros están dados por $D_{i}=\frac{2 v}{f}$ (Expósito-Díaz et al. 2009), considerando la velocidad máxima del giro mayor $\left(0,29 \mathrm{~m} \mathrm{~s}^{-1}\right)$ obtenemos un radio inercial de $5,77 \mathrm{~km}$, mucho más pequeño que el radio del giro ciclónico (140 km, ver tabla 1). Los giros inerciales son forzados por el viento, el que el período inercial sea menor que el rotacional y que el período rotacional sea próximo al del giro afectado por la vorticidad potencial; así como, el que el radio del giro sea mucho mayor que el radio inercial, implica que es un proceso forzado por la conservación de la vorticidad potencial.

\section{Discusión}

Los resultados encontrados en este estudio indican que la conservación de la vorticidad potencial juega un papel importante en la formación del giro ciclónico en la parte este de la bahía, donde el fuerte gradiente de profundidad influye en la modificación del patrón de corrientes. Finalmente, se encuentra que de acuerdo con el valor del número de Rossby el giro ciclónico de la Bahía de Campeche es un giro de gran escala.

Es importante mencionar que los datos analizados, los de la campaña oceanográfica y los de satélite, no son de la misma fecha, pero los resultados mostrados en la Figura 7 concuerdan en las posiciones de los giros ciclónico y el anticiclónico obtenido en ambos casos, lo que sugiere que el giro es una estructura cuasi permanente en la bahía.

Más allá de la importancia que tiene el giro ciclónico de la Bahía de Campeche como proceso hidrodinámico y mecanismo de transporte de diversas propiedades oceánicas, como son la masa y la energía, el giro tiene fuertes implicaciones biológicas, 
como son las corrientes divergentes en la superficie que inducen un ascenso de agua subsuperficial por efecto de su vorticidad positiva. Los movimientos ascendentes pueden transportar nutrientes de las capas subsuperficiales a la capa superficial, que pueden ser usados por el fitoplancton para procesos de fotosíntesis (Morán et al. 2001). La zona central de los giros ciclónicos es altamente turbulenta a escala del tamaño del fitoplancton y los nutrientes no inducen florecimientos importantes en esta región ya que el fitoplancton no puede tomar los nutrientes fácilmente por la alta turbulencia. Al generarse una divergencia por el sentido de la circulación ciclónica del giro, las aguas con altas concentraciones de nutrientes ascienden en el centro del giro y transportan los nutrientes hacia la periferia del mismo, esto último tiende a generar una distribución diferenciada del fitoplancton; es decir en el centro del giro se tiene un predominio de dinoflagelados con menor contenido de clorofila, mientas que en la periferia del giro se presenta un predominio de diatomeas (Coria-Monter et al. 2014). En las regiones periféricas del giro la turbulencia es menos intensa y el fitoplancton puede aprovechar mejor estos nutrientes. En cuanto al zooplancton, se ha observado que el giro ciclónico de la Bahía de Campeche modula la distribución y abundancia de diversas especies (Monreal-Gómez \& Salas-de-León 1990). Salas-de-León et al. (1998) determinaron que antes de la formación del giro ciclónico las corrientes en la bahía son de este a oeste, esto hace que las comunidades de larvas de peces neríticas, oceánicas y neríticas de influencia fluvio-lagunar de la bahía, se distribuyan en forma alargada paralelas a la costa, al formarse el giro éste atrapa organismos que se distribuyen básicamente en la región oceánica, los de ambientes neríticos y fluvio-lagunares son confinados hacia la costa. Al desplazarse el giro de este a oeste se lleva consigo los organismos de ambiente oceánicos mientras que los de ambiente neríticos y fluvio-lagunares son trasportados hacia el este, produciendo una oscilación anual en la distribución y abundancia de los organismos antes mencionados por el efecto del giro. Es importante mencionar que en la parte sur de la Bahía de Campeche desemboca el río Grijalva-Usumacinta, el segundo en caudal del Golfo de México, el primero es el Misisipi. Durante la etapa previa a la formación del giro cuando las corrientes son de este a oeste, las corrientes desplazan la pluma del río Grijalva-Usumacinta hacia el oeste hasta en aproximadamente $30 \mathrm{~km}$, posteriormente cuando el giro está bien formado y se ubica en el centro de la Bahía de Campeche, la pluma es desplazada hacia el este en aproximadamente 40 $\mathrm{km}$, esto produce una oscilación semianual de la pluma con importantes consecuencias ecológicas y sedimentarias en la región (Monreal-Gómez \& Salas-de-León 1990)
Pérez-Brunius et al. (2013) encuentran que el giro ciclónico se extiende por debajo de los $1000 \mathrm{~m}$ y que su forma y posición están delimitados por la topografía de la región, estos autores no encuentran en su estudio la corriente de frontera oeste del Golfo de México reportada por Ichiye (1962), Monreal-Gómez \& Salas-de-León (1990) y Vázquez-de la Cerda et al. (2005), conocida como Corriente Mexicana. Vázquez-de la Cerda et al. (2005) mencionan que la formación de dicha corriente se explica por el balance de vorticidad. En esta investigación se observaron la Corriente Mexicana y el giro ciclónico de la Bahía de Campeche. La formación del giro ciclónico se debe fundamentalmente a una redistribución de la forma de las corrientes inducida por la conservación de vorticidad, cuando el flujo pasa de una zona poco profunda a una profunda, esto en la región este de la bahía. Ambos casos, la formación de las corrientes de frontera oeste y los giros, los explica claramente Stewart (2008) para condiciones generales del océano. La corriente de frontera oeste de la Bahía de Campeche, está modulada por la formación y desplazamiento de los giros ciclónicos (Salas-de-León et al. 1998). El giro se forma, parte del sureste de la Bahía de Campeche y llega hasta el oeste de la bahía donde choca con la costa y se destruye, cuando se forma el giro la Corriente Mexicana se debilita y se invierte.

Vázquez-de la Cerda $(1975,1993)$ describe la circulación ciclónica en la Bahía de Campeche como semipermanente y la atribuye al esfuerzo rotacional del viento; por otro lado, menciona que no hay una clara evidencia de la intensificación hacia el oeste de la circulación ciclónica, implicando que es un giro friccional dominado por el efecto- $\beta$

Sin embargo, los modelos numéricos de Monreal-Gómez \& Salas-de-León (1990) reproducen el giro ciclónico, sin viento, sin fluctuaciones en la Corriente de Yucatán, sin variaciones de la Corriente de Lazo y sin desprendimientos de giros anticiclónicos, mostrando que la formación del giro es una respuesta a la geometría de la cuenca.

\section{Agradecimientos}

Este trabajo estuvo financiado por los proyectos institucionales 144 y 145 del Instituto de Ciencias del Mar y Limnología de la UNAM. Miguel Ángel Díaz Flores agradece al CONACYT por la beca recibida durante sus estudios de doctorado. El tiempo del buque oceanográfico Justo Sierra para la expedición de investigación fue financiado por la Universidad Nacional Autónoma de México (UNAM). Agradecemos a los participantes en este crucero de investigación, incluyendo el capitán y la tripulación. Los autores agradecen los comentarios de los árbitros anónimos que enriquecieron grandemente el escrito. 


\section{LITERATURA CITADA}

Bracco A \& J Pedlosky. 2003. Vortex generation by topography in locally unestable baroclinic flows. Journal of Physical Oceanography 33(1): 207-219.

Behringer DW, RL Molinari \& JF Festa. 1977. The variability of anticyclonic current patterns in the Gulf of Mexico. Journal of Geophysical Research 82(34): 5469-5476.

Cochrane JD. 1963. Yucatan Channel. Annual Report. Department of Oceanography, Texas A\&M University, College Station, Ref. 63-18A: 6-11.

Coria-Monter E, MA Monreal-Gómez, DA Salas-de-León, J Aldeco-Ramírez \& M Merino-Ibarra. 2014. Differential distribution of diatoms and dinoflagellates in a cyclonic eddy confined in the Bay of La Paz, Gulf of California, Journal of Geophysical Research, Oceans 119: 6258-6268, <doi:10.1002/2014JC009916>.

Díaz-Flores MA. 2004. Estudio de las corrientes en la Bahía de Campeche utilizando un perfilador acústico Doppler (ADCP). Tesis de Maestría en Ciencias del Mar y Limnología, UNAM, Cd. de México, 68 pp.

Expósito-Díaz G, DA Salas-de-León, MA Monreal-Gómez, D Salas-Monreal \& F Vázquez-Gutiérrez. 2009. Corrientes inerciales en el sur del Golfo de México. Ciencias Marinas 35(3): 287-296.

Hamilton P, GS Fargion \& DC Biggs. 1999. Loop Current eddy path in the Western Gulf of Mexico. Journal of Physical Oceanography 29: 1080-1207.

Hurlburt HE \& JD Thompson. 1980. A numerical study of loop current intrusions and eddy shedding. Journal of Physical Oceanography 10: 1611-1651.

Hurlburt HE \& JD Thompson. 1982. The dynamics of the loop current and shed eddies in a numerical model of the Gulf of Mexico. In: Nihoul JCJ (ed). Hydrodynamics of semienclosed seas, Proceedings of the 13th International Liege Colloquium on Ocean Hydrodynamics, Elsevier Oceanography Series 34: 243-297.

Ichiye T. 1962. Circulation and water mass distribution in the Gulf of Mexico. Geofísica Internacional 2: 4776.

Leipper DF. 1970. A sequence of current patterns in the Gulf of Mexico. Journal of Geophysical Research 75: 637-657.

Maul GA. 1977. The annual cycle of the gulf loop current, Part II: Observations during a one-year time series. Journal of Marine Research 35: 29-47.

McWilliams. 1985. Submesoescale, coherent vortices in the ocean. Reviews of Geophysics 23(2): 165-182.

Molinari RL, JF Festa \& DW Behringer. 1978. The circulation in the Gulf of Mexico derived from estimated dynamic height fields. Journal of Physical Oceanography 8(6): 987-996.

Monreal-Gómez MA. 1986. Modélisation du mode barotrope et du premier mode barocline dans le Golfe du Mexique.
Tesis de doctorado, Université de Liège, Liège Bélgica, 171 pp.

Monreal-Gómez MA \& DA Salas-de-León. 1990. Simulación de la circulación de la Bahía de Campeche. Geofísica Internacional 29(2): 101-111.

Monreal-Gómez MA \& DA Salas-de-León. 1997. Circulación y estructura termohalina del Golfo de México. En: LavínPeregrina MF (ed). Contribuciones a la oceanografía física en México. Monografía 3: 183-199, Unión Geofísica Mexicana, Cd. de México.

Morán XA, I Tuapier, E Vázquez, S Ruíz, L Arin, P Raimbault \& M Estrada. 2001. Physical-biological coupling in the Algerian Basin (SW Mediterranean): influence of mesoscale instabilities on the biomass and production of phytoplankton and bacterioplankton. Deep-Sea Research 48: 405-437.

Nowlin WD. 1972. Winter circulation patterns and property distributions. In: Capurro LRA \& JL Reid (eds). Contributions on the physical oceanography of the Gulf of Mexico, Texas A\&M University Oceanographic Studies 2: 3-15. Gulf Publishing Company, Houston.

OhImann JC, PP Niiler, CA Fox \& RR Leben. 2001. Eddy energy and shelf interactions in the Gulf of Mexico. Journal of Geophysical Research 106(C2): 2605-2620.

Pérez-Brunius P, P García-Carrillo, J Dubranna, J Sheinbaum \& J Candela. 2013. Direct observations of the upper layer circulation in the southern Gulf of Mexico. Deep-Sea Research II 85: 182-194.

Salas-de-León DA, MA Monreal-Gómez, L SanvicenteAñorve \& C Flores-Coto. 1998. Influence de la circulation â long terme sur la répartition des organismes zooplanctoniques dans la Baie de Campeche, Mexique. Oceanologica Acta 21(1): 87-93.

Smith DC IV. 1986. A numerical study of loop current eddy interaction with topography in the Western Gulf of Mexico. Journal of Physical Oceanography 16: 1260-1272.

Stewart RH. 2008. Introduction to physical oceanography. Department of Oceanography, Texas A\&M University. <http://www.colorado.edu/oclab/sites/default/files/attachedfiles/stewart_textbook.pdf>

Sturges W \& A Bozec. 2013. On a puzzling disagreement between observations and numerical models in the Central Gulf of Mexico, Journal of Physical Oceanography 43: 2673 2681. <doi:10.1175/JPO-D-13-081.1>

Sturges W \& JC Evans. 1983. On the variability of the loop current in the Gulf of Mexico. Journal of Marine Research 41:639-653.

Vázquez-de la Cerda AM. 1975. Currents and Waters of the upper 1200 meters of the Southwestern Gulf of México. Master's Thesis, Texas A\&M University, College Station, $107 \mathrm{pp}$. 
Vázquez-de la Cerda AM. 1993. Bay of Campeche Cyclone. Tesis de Doctorado en Ciencias, Texas A\&M University, College Station, $91 \mathrm{pp}$.

Vázquez-de la Cerda AM, RO Reid, SF DiMarco \& AE Jochens. 2005. Bay of Campeche Circulation: An update. In: Sturges W \& ALugo-Fernández (eds). Circulation in the Gulf of Mexico: Observations and models, pp. 279-293. American Geophysical Union, Washington. <doi: 10.1029/ 161GM20>

Velasco-Mendoza H. 1994. Modelo numérico de circulación para la Bahía de Campeche. Tesis de Maestría en Ciencias del Mar, Instituto de Ciencias del Mar, UNAM, Cd. de México, 133 pp.

Vidal MVV, FV Vidal, AF Hernández, E Meza \& JM PérezMolero. 1994. Baroclinic flows, transports, and kinematic properties in a cyclonic-anticyclonic-cyclonic ring triad in the Gulf of Mexico. Journal of Geophysical Research 99(C4): 7571-7597.
Villanueva EE, VM Mendoza \& J Adem. 2002. Some numerical solutions of the barotropic vorticity equation applied to Gulf of Mexico. Atmósfera 15: 173-184.

Vukovich FM \& GA Maul. 1985. Cyclonic eddies in the eastern Gulf of Mexico. Journal of Physical Oceanography 15: 105-117.

Vukovich FM, BW Crissman, M Bushnell \& WJ King. 1979. Some aspects of the oceanography of the Gulf of Mexico using satellite and in situ data. Journal of Geophysical Research 84(C12): 7749-7768.

Recibido el 11 de abril de 2016 y aceptado el 22 de junio de 2017

Editor: Claudia Bustos D / col. Dra. Paola Dávila 